1C.003 PUBLIC HEALTH POLICY AND FALLS PREVENTION AMONG OLDER PEOPLE IN THE COMMUNITY

${ }^{1,2}$ Aleksandra Natora*, ${ }^{1}$ Jennie Oxley, ${ }^{1}$ Terry Haines, ${ }^{1}$ Linda Barclay, ${ }^{2}$ Bruce Bolam. ${ }^{1}$ Monash University, Melbourne, Australia; ${ }^{2}$ Department of Health and Human Services Victoria, Melbourne, Australia

10.1136/injuryprev-2021-safety. 10

Background Globally, falls are a leading cause of injury, hospitalisation, disability and death among older people, especially those living in private homes. Public health policy can be a successful population level strategy for injury prevention, however there is a lack of evidence of its impact on incidence rates of falls-related hospitalisations or deaths.

Methods A policy analysis was conducted through a systematic literature review of effectiveness of international public health policy on falls prevention in the community by government jurisdictions for the period 2007 to 2019, using the World Health Organization's 2007 Global Report on Falls in Older Age as a starting point. Falls prevention policy documents were systematically identified via literature databases and government websites, and were included if they focussed on community-dwelling older people, and if they indicated pre- and post-policy incidence rates of falls-related hospitalisations or deaths.

Results This presentation will provide a synthesis of evidence of the effectiveness of public health policy interventions on falls prevention in the community to stimulate audience conversation and insight into international examples of falls prevention policy. The synthesis will be thematic and narrative.

Conclusions Public health policy is an important population level intervention for falls prevention in the community, however its impact on incidence rates of falls-related hospitalisations and deaths is variable.

Learning Outcomes This presentation will allow insights into international policy approaches to falls prevention in the community which may inform future government policy efforts.

\section{C.004 EPIDEMIOLOGY OF FATAL HOME INJURIES IN RURAL BANGLADESH}

1,2Shumona Salam*, ${ }^{2}$ Mehedi Hasan, ${ }^{2}$ Qazi Sadeq-ur Rahman, ${ }^{2}$ rrteja Islam, ${ }^{2}$ Shams El Arifeen. ${ }^{1}$ The University of Sheffield, Sheffield, UK; ${ }^{2}{ }^{1} \mathrm{cddr}, \mathrm{b}$, Dhaka, Bangladesh

10.1136/injuryprev-2021-safety. 11

Background The home is the most common location for fatal injuries in Bangladesh with $60 \%$ of deaths occurring in and around the home. This paper describes the epidemiology of fatal home injuries in rural Bangladesh

Methods A census and baseline survey was conducted as part of the Saving of Lives from Drowning (SoLiD) project in 7 purposively sampled rural sub-districts of Bangladesh. Information on socio-demographic characteristics, injury mortality and morbidity were collected from 1.2 million people between June and November, 2013. Descriptive analyses were done to quantify the burden of unintentional fatal home injuries.
Results 267 injury deaths were recorded over the 1-year recall period in the home environment (mortality rate was $23[95 \%$ CI 20-26] per 100000 population per year). Leading causes of home injury deaths were drowning (53.7\%), suicide (10.8\%), falls (12.3\%) and burns (9.3\%). Children aged 1-4 years (97 [95\% CI 78-120] per 100000) and adults older than 65 years (81 [95\% CI 62-105] per 100000) were at high risk for injuries in the home $(\mathrm{p}<0.000)$. Drowning was the leading cause of death among children aged 1-9 years. Suicide followed by drowning was the main causes of deaths among adolescent males aged 10-14 years. Suicide followed by burn was the main cause of death among females aged 15-24 years. Falls was the main cause of death among adults older than 65 years.

Recommendation Interventions need to be promoted to ensure safer behaviours and help change home environments for families especially children in rural Bangladesh.

\section{C.005 USING ONLINE PARENTING DISCUSSION FORUMS FOR UNDERSTANDING FALL INJURY MECHANISMS IN INFANTS}

${ }^{1,2}$ Nipuna Cooray*, ' ${ }^{1}$ Si Sun, ${ }^{3}$ Susan Adams, ${ }^{1,2}$ Lisa Keay, ${ }^{4}$ Natasha Nassar, ${ }^{1,2}$ Julie Brown. ${ }^{1}$ The George Institute for Global Health, Newtown, Australia; ' University of New South Wales, Sydney, Australia; ${ }^{3}$ Sydney Children's Hospital, Randwick, Australia; ${ }^{4}$ University of Sydney, Sydney, Australia

10.1136/injuryprev-2021-safety. 12

Background Falls are the most common mechanism of injury in infants and may result in traumatic brain injuries. Most previous studies fail to focus on fall types specific to infants who are mostly not independently mobile, and lack contextual information vital to development of preventive interventions. Online parenting forums provide an unobtrusive rich data source where infant fall incidents are commonly discussed. The aim of this study was to evaluate online parenting forums to gain insight into events leading to infant falls.

Method Fall incident discussions on an Australian parenting website were identified and downloaded using site-specific google search queries and a programming script. A qualitative descriptive approach was used to analyse and categorise contextual information into 'pre-cursor events' and 'influencing factors' for infant falls.

Results Of the 461 infant fall incidents identified, common mechanisms were falls from furniture and when being carried or supported by someone, falls from baby products and falls on the same level. Common precursor events were infant rolling off or left alone on furniture, product misuse, adults falling asleep while holding the infant; and tripping/slipping while carrying the infant. Common influencing factors were rapid infant motor development, lapses in caretaker attention and trip hazards.

Discussion and Conclusions This study provides insight into parental behaviours preceding infant falls. Findings will help define target behaviours requiring modification and inform development of targeted interventions to prevent falls in infants.

Learning Outcomes Online parenting forums can be utilised to explore child injury scenarios that are vital for intervention development. 